\title{
Sub-Tenon's anaesthesia: complications and their prevention
}

\begin{abstract}
The advent of a new technique that is considered much safer than previously established one leads to its rapid adoption. This usually leads to the identification of previously unreported complications of the new technique, and a re-assessment of its position in clinical care, which is precisely the state of play with the sub-Tenon's block. The sub-Tenon's block was introduced into the clinical practice in early 1990. A systematic recent search of subject headings such as complications of sub-Tenon's block, subtenon, orbital block, orbital block complications, and orbital anaesthesia was performed in Medline, EMBASE, and Cochrane database. Indeed there are complications of sub-Tenon's block published as case reports and the exact incidence of these complications is not known. Management and preventive measures of these complications are described. Although the sub-Tenon's block appears to be relatively

safer than needle-based blocks but a proper

prospective, randomized, double-blind controlled trial is essential for scientific proof that sub-Tenon's block is better than needlebased blocks.

Eye (2011) 25, 694-703; doi:10.1038/eye.2011.69; published online 1 April 2011
\end{abstract}

${ }^{1}$ Department of Anaesthesia, Khoo Teck Puat Hospital, Singapore

${ }^{2}$ The James Cook University Hospital, Middlesbrough, UK

Correspondence:

CM Kumar, Department of Anaesthesia, Khoo Teck Puat Hospital, 90 Yishun Central, Singapore 768828, Singapore

Tel: + 656602 2317;

Fax: + 6566023648

E-mail: chandra.kumar2406 @gmail.com

Received: 15 November 2010

Accepted in revised form: 27 February 2011 Published online: 1 April 2011
Keywords: sub-Tenon; ophthalmic regional anaesthesia; ophthalmic blocks; eye blocks; complications of ophthalmic block; complications

\section{Introduction}

The majority of ophthalmic surgical procedures in adults are performed under local anaesthesia depending on surgical procedure, surgeon, and patients. ${ }^{1,2}$ Cataract extraction with lens implant is the most frequently performed surgery. Some but technique, choice, and preferences vary
CM Kumar ${ }^{1}, \mathrm{H}^{\mathrm{Eid}}{ }^{2}$ and C Dodds ${ }^{2}$

surgeons perform this surgery in selected patients under topical anaesthesia but complicated cataract and other procedures may require intraconal (retrobulbar), extraconal (peribulbar), and sub-Tenon's blocks, which provide akinesia as well as anaesthesia. ${ }^{3-7}$

One or more minor complications occur in $4.3 \%$ of 38058 all local ophthalmic blocks. ${ }^{5}$ Serious sight- and life-threatening events occurred in 25 eyes $(0.066 \%)$, undergoing needle or sub-Tenon's blocks but minor complications were 2.3 times more common with sub-Tenon's blocks. The needle blocks had a 2.5 -fold increased risk of serious sight- and lifethreatening complications (Table 1$)^{8-14}$ and their popularity has declined.

The sub-Tenon's was introduced as a simple, safe, and effective block in the early 1990s. ${ }^{15-18}$ In the sub-Tenon's block, a careful dissection is performed, usually in the inferonasal quadrant, to gain access to the space under the Tenon's capsule, and then a blunt sub-Tenon's cannula is inserted and local anaesthetic agent of choice is injected. ${ }^{15-17}$ A variety of sub-Tenon's cannulae ${ }^{19-23}$ are available but a $2.54 \mathrm{~cm}$ long, blunt, and curved posterior metal sub-Tenon's cannula described by Stevens ${ }^{16}$ is the most commonly used. This block has become popular in many countries including the United Kingdom and a recent study indicated that $>87.8 \%$ members of the British Ophthalmic Anaesthesia Society use this technique in their routine practice. ${ }^{24}$ The sub-Tenon's block is probably popular because it provides good akinesia, ${ }^{25}$ better intraoperative analgesia, and operating conditions compared with needle blocks and a $50 \%$ reduction in posterior capsular tear/vitreous loss compared with topical anaesthesia. ${ }^{26}$

Although performance of a sub-Tenon's block requires some dexterity in the handling of surgical instruments but most practitioners develop competence after about 60 blocks. ${ }^{27,28}$ The sub-Tenon's block is used for cataract 


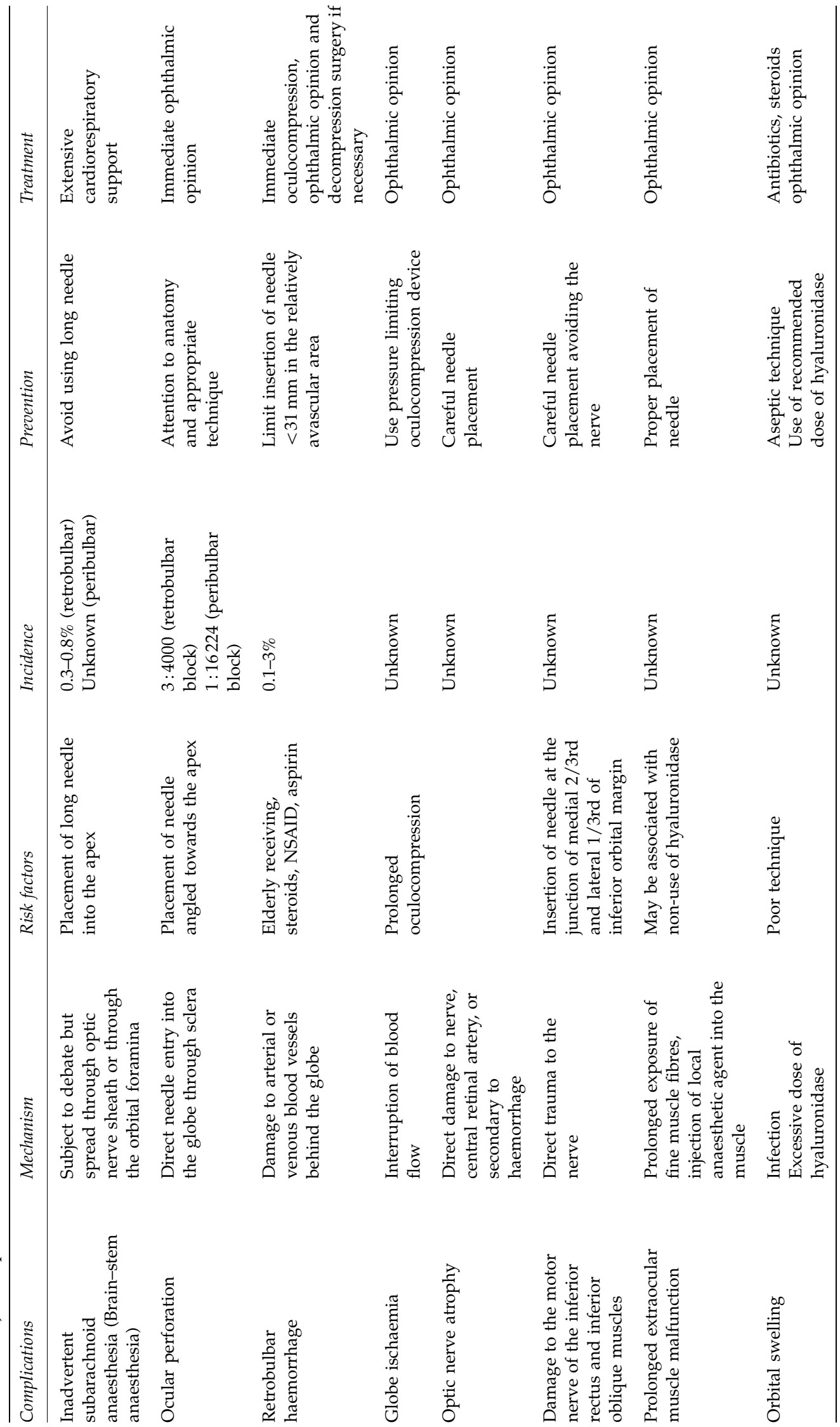


surgery, strabismus surgery, vitreoretinal surgery, optic nerve fenestration, delivery of drugs around the globe, and postoperative pain relief (Table 2). ${ }^{29,30}$

Although the technique is considered relatively safe and its popularity is increased, case reports of minor as well as sight- and life-threatening complications have been reported. We performed a systematic search of the Medline, EMBASE, and Cochrane databases till October 2010. The combinations of the following medical subject headings: orbital anaesthesia, eye blocks, cataract, vitreoretinal surgery, strabismus surgery, subtenon, sub-Tenon's, complications of orbital block, and complications of orbital anaesthesia were searched. References and citations were excluded if they were nonEnglish language. Most complications were reported as case reports and a meta-analysis was not feasible. This article reviews major sight- and life-threatening complications of sub-Tenon's block (Table 3), their possible aetiology, risk factors, prevention, and management.

\section{Minor complications}

Minor complications such as pain, chemosis, and subconjunctival haemorrhage are not sight threatening and although they occur with fairly high frequency they do not usually interfere with surgery.

\section{Pain}

The injection of a local anaesthetic solution can produce a mild stinging or burning sensation and can also cause a feeling of pressure because of widening and stretching of the potential sub-Tenon's space. ${ }^{30}$

The incidence of pain during sub-Tenon's injection with posterior metal cannula is reported in up to $44 \%$ of patients. ${ }^{16}$ Pain scores on a visual analogue scale

Table 2 Limitations and relative contraindications of sub-Tenon's block (modified from Kumar and Dodds) ${ }^{30}$

\section{Limitations}

Previous sub-Tenon's block in the same quadrant

Previous extensive vitreo-retinal surgery

Previous repeated strabismus surgery

Eye trauma

Infection to the orbit

Relative contraindications

Severe ocular pemphigoid

Surgery requiring complete akinesia (viscacanalstomy) Surgery where chemosis and subconjunctival haemorrhage may compromise the outcome of surgery (glaucoma filtration surgery)
$(0=$ no pain, $10=$ worst imaginable $)$ have been reported as high as 5 and smaller cannulae appear to offer a marginal benefit. ${ }^{31}$ In the Auckland Hospital study ${ }^{32}$ of 6000 patients who received sub-Tenon's block, the incidence of pain during injection was 32 with $7 \%$ of patients experiencing more than mild discomfort, $7 \%$ of patients also complained of some discomfort during surgery. The surgeon and patient acceptability rate was 98.8\%.

Pain on injection can be reduced by good surface anaesthesia, gentle insertion of the cannula, and slow injection. As with all regional anaesthetic techniques, there must be continuous explanation and reassurance to the patient according to the guidelines published by The Royal College Anaesthetists and the Royal College of Ophthalmologists in 2001. ${ }^{33}$ The common practice of warming the local anaesthetic solution before subTenon's block was not found to decrease pain experienced during injection. ${ }^{34}$ The range of ophthalmic operations that can be safely managed using a sub-Tenon's block is usually associated with a minimal need for postoperative analgesics. ${ }^{35}$

\section{Chemosis}

Chemosis is a swelling or ballooning of the conjunctiva and signifies spread of the local anaesthetic anteriorly from the sub-Tenon's space during injection or an incorrect injection into the subconjunctival space rather than the sub-Tenon's space.

The incidence of chemosis with posterior metal cannula is $23 \%{ }^{15}$ and increases to $100 \%^{31}$ with shorter cannulae. Chemosis is more likely to occur if dissection of the Tenon's capsule is inadequate, or if a large volume of local anaesthetic is injected. Chemosis may be confined to the site of injection or extend to other quadrants. ${ }^{31}$ It does not usually interfere with cataract surgery but can compromise the operative field especially during glaucoma surgery.

Careful dissection and applying gentle pressure on the globe after sub-Tenon's block may reduce the swelling and limit its expansion. ${ }^{30}$

\section{Subconjunctival haemorrhage}

Subconjunctival haemorrhage is usually due to the tearing of small blood vessels during conjunctival or Tenon capsule dissection. The haemorrhage is usually limited to the area of dissection but can spread to other quadrants. $^{36}$

A red looking eye may also be due to conjunctival vasodilatation following drug instillation. The incidence of red eye following sub-Tenon's block varies from $7.4-100 \%, 31,32$ being higher with shorter cannulae. 


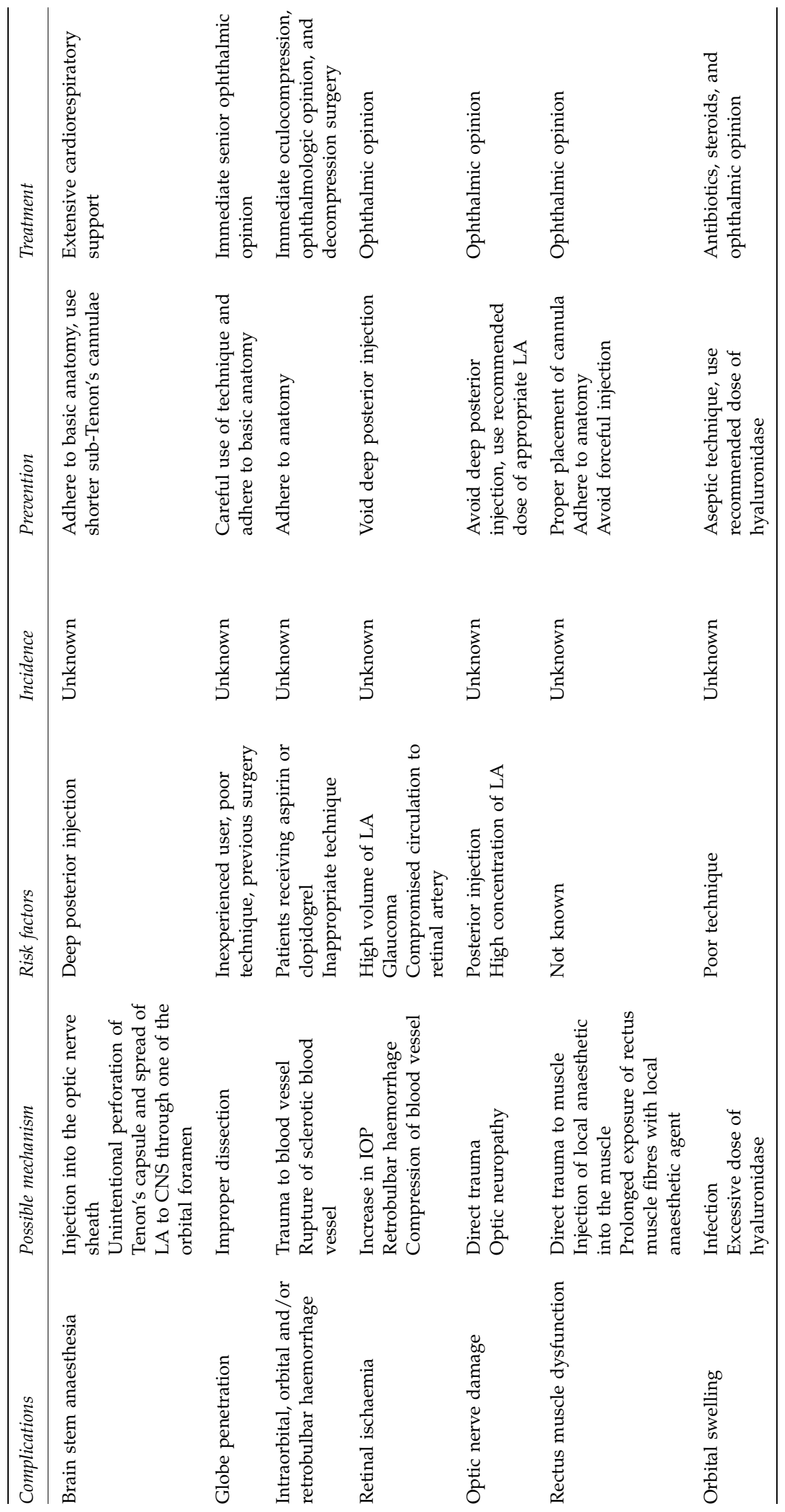


The lower incidence reported could be due to the exclusion of patients who had red looking eyes because of conjunctival injection or the subjective assessment. An objective method to assess conjunctival haemorrhage using comparison of photographs has been proposed ${ }^{37}$ but may not be clinically feasible.

Patients taking antiplatelet agents, such as aspirin and clopidogrel, appear to have a higher incidence of subconjunctival haemorrhage, although this is not statistically significant. ${ }^{38}$ The current recommendation is to continue anticoagulant medications and maintain the international normalized ratio within the appropriate therapeutic range before ophthalmic surgery. ${ }^{39,40}$ Continuation of aspirin did not cause difficulty in stopping the bleeding in any individual case and discontinuation of the medication had no effect on the intraoperative course or postoperative outcome. ${ }^{41}$ In the Auckland Hospital series, surgery was abandoned in 1 of the 6000 patients because of a large subconjunctival haemorrhage. ${ }^{32}$ However, this was not sight threatening and the patient was not taking any anticoagulant medication.

Subconjunctival haemorrhage may be minimized by careful dissection, which reduces the damage to fine vessels. Gentle pressure and application of vasoconstrictor soaked cotton buds may limit the spread of haemorrhage. ${ }^{14}$ Localized conjunctival cautery under microscopic control by ophthalmologists before tissue dissection may significantly reduce the frequency of subconjunctival haemorrhage. ${ }^{42}$ However, no such benefit was obtained when cautery was used by the anaesthetists. ${ }^{43}$ The incidence of this common minor complication is unpredictable and all patients receiving sub-Tenon's block should be informed that the eye might look red in the immediate postoperative period.

\section{Major life- and sight-threatening complications}

\section{Brain stem anaesthesia}

Two cases of possible brain stem anaesthesia after sub-Tenon's block have been reported. The exact aetiology of spread of local anaesthetic agent to the central nervous system is not very well understood. Although the mechanism in these cases is not clear but direct spread of local anaesthetic into the subarachnoid space may have been the cause of brain stem anaesthesia. Rüschen et $a l^{44}$ suggested that local anaesthetic could have crossed the optic nerve dural sheath through a perforation that occurred during dissection of the sub-Tenon's space with the tip of the Westcott scissors. Kumar and Dodds ${ }^{30}$ offer alternative explanations. According to a textbook of anatomy, ${ }^{45}$ the space under the Tenon's capsule is a lymph space, and this follows the optic nerve and continues with the subarachnoid space. Accidental injection of local anaesthetic agent into this part of the posterior sub-Tenon's space can then enter the subarachnoid space through the optic nerve sheath. Unintentional perforation of the Tenon's capsule during injection, subsequent deposition of local anaesthetic into the intraconal space and spread to the central nervous system through an orbital foramen is another possibility. $^{30}$

Symptoms and signs of brain stem anaesthesia range from drowsiness, confusion, and or loss of verbal contact, to cranial nerve palsies, convulsions, respiratory depression, and cardiac arrest. Rüschen et al ${ }^{44}$ reported a patient who became unresponsive to verbal command for nearly $3 \mathrm{~h}$ after a sub-Tenon's block. Fortunately respiration and cardiovascular parameters were stable during the operation and immediate post recovery period. The patient made a full recovery with no neurological deficits. Quantock and Goswami ${ }^{46}$ described a patient who developed a generalized tonicclonic seizure and refractory ventricular fibrillation one minute after sub-Tenon's block injection. Subsequent resuscitation was unsuccessful. Post-mortem examination revealed severe coronary artery disease and the mortality was considered primarily cardiac in origin; however, the possibility of brainstem anaesthesia could not be ruled out.

Immediate availability of trained personnel who can maintain airway and oxygenation is essential. These patients may require careful intravenous fluid therapy and vasopressor agents.

\section{Globe perforation}

Three cases of globe perforation have been reported, two identified at the time of the performance of the block and one 5 weeks later.

Frieman and Friedberg ${ }^{47}$ reported an episode of globe perforation that occurred in a patient with scleral scarring resulting from previous retinal detachment surgery. Incisions were made with sharp scissors to relieve the adhesions but the surgeon continued to advance the scissors more posteriorly until he suddenly felt a dramatic decline in resistance because of globe perforation. Prolonged management of this perforation was required but no adverse visual sequale were reported. Another case of globe perforation was reported which occurred when a metal sub-Tenon's cannula entered the globe through the sclera. ${ }^{48}$

A case of delayed scleral perforation was reported from the United Kingdom ${ }^{44}$ where the sub-Tenon's block was performed by the operating surgeon immediately before the start of cataract surgery. Three $\mathrm{ml}$ of $0.5 \%$ bupivacaine was used for the sub-Tenon's block. The 
patient felt discomfort during the latter part of the operation and was squeezing her eye. This was described as ineffective sub-Tenon's block, which made the operation increasingly difficult. A posterior capsular tear occurred requiring a complex anterior vitrectomy. Five weeks later on routine follow-up, an inferonasal scar attributed to a cannula entry was identified. This case highlights the importance of waiting to achieve an effective block particularly when drugs with a relatively slow onset of action are used.

An urgent ophthalmological opinion must be sought as immediate surgery may limit the damage.

\section{Orbital and/or retrobulbar haemorrhage}

Cases of intraocular, orbital, and retrobulbar haemorrhages of unknown aetiology have been reported. Rüschen et $a l^{44}$ reported two patients who developed hyphaema after the administration of sub-Tenon's block before surgery. Ultrasound examination showed posterior vitreous detachment. Neither patient had the known risk factors for hyphaema, and in both, sub-Tenon's block was followed by ocular massage. Ocular massage with fingers is sometimes performed to aid the spread of the local anaesthetic or reduce oedema formation although a compression device such as the Honan's balloon is more commonly used. However, pressure generated by digital massage can produce a peak intraocular pressure (IOP) of up to $400 \mathrm{~mm} \mathrm{Hg}$, with considerable globe distortion and shearing forces on the ciliary arteries. $^{49}$

There have been three published case reports of orbital and/or retrobulbar haemorrhage occurring between administration of the anaesthetic block and the start of the procedure..$^{50-52}$ There was no visible source of bleeding in one patient ${ }^{51}$ and one other patient was on regular aspirin and clopidogrel. ${ }^{52}$

The causes of intraorbital, orbital, and/or retrobulbar haemorrhage are not clear but direct trauma to blood vessels by the cannula cannot be ruled out. The temporal vortex vein reflects on to the inner surface of the Tenon's capsule posterior to the insertion of the inferior rectus muscle. ${ }^{45}$ The inferonasal quadrant has no such vein loop on the inner Tenon's capsule. However, it is possible that the long metal sub-Tenon's cannula was inserted too far posteriorly. This, coupled with anatomical variations or slight inferior displacement of the cannula during injection, might have led to rupture of the vein and a subsequent retrobulbar haemorrhage. Displacement and rupture of a sclerotic blood vessel because of infusion of fluid behind the orbit was another possibility. ${ }^{51}$

A retrobulbar haemorrhage should be suspected when the globe becomes proptosed over a period of a few minutes. Lid swelling and conjunctival blood vessel engorgement occur. IOP should be determined which is dramatically increased and the retinal circulation should be checked.

Treatment if necessary is directed towards reducing compartment pressure, thus reducing IOP and minimizing the effects on retinal circulation. Immediate oculocompression can be helpful in limiting the severity and extent of haemorrhage. The ophthalmologist should be consulted immediately to evaluate the degree of haemorrhage and for further interventions.

\section{Retinal ischaemia}

Closures of the central retinal vessels have been reported with $^{53,54}$ or without retrobulbar haemorrhage. ${ }^{55}$ Two cases of transient central retinal artery occlusion and another case of permanent loss of vision have been reported after sub-Tenon's block. ${ }^{56}$ One patient in Ruschen et al series ${ }^{44}$ suffered from irreversible visual loss of elusive aetiology and the possibly of a central retinal artery occlusion could not be ruled out. This patient gave a history of transient blindness 6 months earlier presumably caused by arterial spasm and this was considered to be a predisposing factor to the arterial occlusion following sub-Tenon's block. Recently, another episode of retinal arterial occlusion associated with subTenon's block has been reported. ${ }^{57}$

The exact cause of the above complication following sub-Tenon's block is unknown. It could presumably result from an increase in IOP exceeding the mean arterial pressure in the ophthalmic or central retinal artery, or because of increased IOP, which may interfere with the small vessels supplying the optic nerve. ${ }^{57}$ However, the sub-Tenon's block does not appear to be associated with a significant increase in the IOP. ${ }^{58}$ The manipulation of the globe may compromise the retinal perfusion because of spikes in IOP. All orbital blocks including sub-Tenon's cause a marked decrease in pulsatile ocular blood flow, persisting at least for $10 \mathrm{~min}$ after the injection. ${ }^{59,60}$ It is hypothesized that the decrease in ocular blood flow is the result of vasoconstriction caused by the high local concentration of the local anaesthetic drug. ${ }^{60}$ Inhibition of endothelium-dependent relaxation of the ciliary arteries or anaesthetic blockade of the vasodilator nerves are other possible explanation for vasoconstriction. ${ }^{61}$

Although the risk of retinal ischaemia after local anaesthetic injection is very small but it deserves consideration in patients with compromised ocular circulation or significant glaucomatous optic neuropathy.

Signs and symptoms of retinal artery occlusions are usually late. Retinal arterial occlusion is an ophthalmic emergency and prompt treatment is essential.

Nonspecific methods to increase blood flow and dislodge 
emboli include digital massage, intravenous acetazolamide, and methylprednisolone. Additional measures include paracentesis of aqueous humour to decrease IOP acutely. Pan-retinal photocoagulation with an argon laser may be effective in early stages.

\section{Optic nerve damage}

There has been one case report of a patient who developed total blindness after cataract extraction possibly owing to optic neuropathy resulting from direct trauma by the tip of the sub-Tenon's cannula. ${ }^{62}$ The investigators demonstrated, on cadaveric eyes, that the tip of the metal sub-Tenon's cannula (22-26 mm long) could reach the optic nerve when the cannula was advanced along the sclera in the inferonasal quadrant. The use of $4 \%$ lidocaine causing neurotoxicity might be another possibility.

\section{Rectus muscle dysfunction}

There have been several published reports of diplopia associated with sub-Tenon's block and the inferior rectus is the most commonly affected muscle.

Jaycock et $a l^{63}$ described three cases of persistent inferior rectus muscle restriction after sub-Tenon's block provided via the inferonasal quadrant. The investigators believed that direct trauma to the muscle with haematoma formation occurred at the time of the sub-Tenon's block with subsequent contracture of the affected muscle. All patients suffered from immediate periorbital bruising. Two of these patients received a local anaesthetic mixture of lidocaine $4 \%$ and bupivacaine $0.75 \%$, which might have contributed to myotoxicity. Three more cases of inferior rectus muscle restriction were reported by Adams et al. ${ }^{64}$ Similarly, Merino et al ${ }^{65}$ reported eight patients who developed restrictive strabismus after sub-Tenon's block. Strabismus surgery was required in four cases. Lignocaine 5\% was the anaesthetic agent used for the sub-Tenon's block.

Spierer and Schwalb ${ }^{66}$ reported transient diplopia resulting from superior oblique muscle paresis after cataract surgery. A sub-Tenon's block was performed in the superotemporal quadrant using a metal cannula that was advanced to its fullest extent along the sclera. Spontaneous recovery ensued (within one month).

Muscle dysfunction after sub-Tenon's block may result from direct trauma to the muscle with either the scissors or trauma by the rigid metal cannula ${ }^{63,64}$ or from direct myotoxicity of the local anaesthetic solution.

Conventional local anaesthetic concentrations such as $2 \%$ lignocaine, $0.75 \%$ bupivacaine, levobupivacaine, or $1 \%$ ropivacaine are in common use. Higher concentrations may be neurotoxic as well as myotoxic and should be avoided. Inflammation or infection introduced into the sub-Tenon's space leading to adhesion formation may be another mechanism of muscle injury. ${ }^{64}$

Muscle injury may not be the only cause of diplopia after cataract surgery. The prolonged disruption of binocular vision and the abrupt change in the sensory situation after the cataract operation with lens implantation may lead to strabismus or deterioration of a pre-existing strabismus. ${ }^{64}$ Evaluation of binocular vision and eye movements before cataract operation may be helpful in diagnosis and management of diplopia after surgery.

Care is required during dissection and insertion of metal cannula during the block. Once diagnosed, the case should be treated by strabismus surgeon for corrective surgery.

\section{Orbital swelling}

Several cases of proptosis developing within the first few days after surgery under sub-Tenon's block have been reported. ${ }^{67-73}$

Possible mechanisms included infection, hyaluronidase-induced orbital inflammation, or reaction to anaesthetic agents. None of the patients had appropriate tests for allergic reactions in the reported cases. At the time these complications were reported, instillation of conjunctival povidone iodine was not applied before sub-Tenon's block, as the common practice then was to apply povidone iodine only after sub-Tenon's block and before surgery. Some of these patients had high doses of hyaluronidase 500-1500 IU added to the injected local anaesthetic. ${ }^{71}$

The severity of the manifestations varied and most patients were afebrile with normal white blood cell count. Oral and systemic antibiotics with or without steroid resolved the problems. One patient received an oral antihistamine. The orbital swellings resolved within 2-4 weeks in all cases. Only two cases of orbital swelling had clinical picture of infection associated with fever, purulent discharge, and positive cultures. The first patient presented with a catastrophic endophthalmitis and orbital cellulitis that resulted in loss of vision, following uncomplicated cataract surgery under sub-Tenon's block. ${ }^{67}$ In the second case, orbital cellulitis developed in an immunologically compromised patient who presented with inferonasal corneal perforation. ${ }^{70}$ The patient had undergone a corneal gluing procedure under sub-Tenon's block. The orbital cellulitis resolved after intravenous antibiotics.

The safety of providing sub-Tenon's block in the presence of active corneal ulceration and altered 
conjunctival flora is not known. If sub-Tenon's block is required, extreme caution is necessary. Corneal gluing can be performed under topical anaesthesia and sub-Tenon's block should be avoided.

Hyaluronidase-induced orbital inflammation (which has also been reported after needle blocks) should be considered when a patient presents with a rapid onset of orbital swelling, especially in the presence of previous exposure to hyaluronidase. ${ }^{74}$ Hyaluronidase-associated orbital inflammation may predispose the eye to infection. $^{73}$

Sub-Tenon's block, is a 'no-touch' technique and routine aseptic precautions should be practised. It does involve a significant breach of the conjunctiva that could potentially serve as an access for pathogens into the orbital cavity. Instillation of specially formulated povidone iodine $5 \%$ into the conjunctival sac before injection is recommended before opening up the conjunctiva. ${ }^{75}$ Excessive dose of hyaluronidase should be avoided and must not exceed $>15 \mathrm{IU} / \mathrm{ml}^{76}$

Early recognition and treatment of postoperative orbital inflammation by steroids and appropriate antibiotics may prevent potentially serious visual consequences.

\section{Other non-sight and non-life threatening}

Eye lashes ${ }^{77,78}$ and intact subconjunctival insect entrapment ${ }^{79}$ after sub-Tenon's block have been described. A large conjunctival incision that is sited too anteriorly can rarely create a potential entry site for unwanted foreign bodies may also be associated with inward folding of a conjunctival flap resulting in inclusion cyst formation. ${ }^{80}$

Care should be taken when administering a subTenon's block to ensure that the conjunctival incision is small and sited correctly. Vigilance is needed in patients reporting a persistent foreign body sensation or eye redness following sub-Tenon's block.

\section{Other untoward medical events that may occur during} sub-Tenon's block

Serious systemic adverse events may occur during anaesthesia and surgery, which are not related to sub-Tenon's block. Five patients in the Auckland study ${ }^{32}$ had panic attacks or cardiovascular compromise and five other patients required therapeutic in terventions for cardiovascular events that occurred during or shortly after sub-Tenon's block.

Routine intraoperative monitoring and vigilance is required to diagnose these events so that appropriate treatment can be instituted immediately.

\section{The ways forward}

Sub-Tenon's block has now been associated with most of the commonly described complications, which are associated with needle blocks but the incidence and nature of these complications appear to be less frequent. The sub-Tenon's block is accepted as a safer alternative. ${ }^{32}$ A recent large UK survey found a lower rate of reported serious complications with sub-Tenon's block compared with retrobulbar and peribulbar techniques. ${ }^{5}$ Although the reasons for some of the complications are not clear but most sight- and life-threatening complications appear to be related to inappropriate or poor technique. Although the type of cannula used was not always specified in the reported cases but it appeared that all the complications occurred while the rigid metal posterior cannula was used. It is surmised, although not proven, that a shorter, less rigid or flexible cannula, and lower volume of local anaesthetic agent can improve the safety profile of sub-Tenon's block. ${ }^{81}$

\section{Conclusion}

The sub-Tenon's block appears to be relatively safer than needle-based blocks but is not without complications and a proper prospective, randomized, double-blind controlled trial is essential for scientific proof. A thorough knowledge of orbital anatomy, attention to the technique, awareness of possible complications, and preventive measures are essential if this technique is to be used safely.

\section{Conflict of interest}

The authors declare no conflict of interest.

\section{Acknowledgements}

This work was supported by the Department of Anaesthesia and Ophthalmology, The James Cook University Hospital, Middlesbrough, UK.

\section{References}

1 Friedman DS, Reeves SW, Bass EB, Lubomski LH, Fleisher LA, Schein OD. Patient preferences for anaesthesia management during cataract surgery. Br J Ophthalmol 2004; 88: 333-335.

2 Ruschen H, Celaschi D, Bunce C, Carr C. Randomised controlled trial of sub-Tenon's block vs topical anaesthesia for cataract surgery: a comparison of patient satisfaction. Br J Ophthalmol 2005; 89: 291-293.

3 Malik A, Fletcher EC, Chong V, Dasan J. Local anesthesia for cataract surgery. J Cataract Refract Surg 2010; 36: 133-152. 
4 Leaming DV. Practice styles and preferences of ARCRS members: 2003 survey. J Cataract Refract Surg 2004; 30: 892-900.

5 El-Hindy N, Johnston RL, Jaycock P, Eke T, Braga AJ, Tole $\mathrm{DM}$ et al. The Cataract National Dataset Electronic MultiCentre Audit of 55567 operations: anaesthetic techniques and complications. Eye 2009; 23: 50-55.

6 Srinivasan S, Fern AI, Selvaraj S, Hasan S. Randomized double-blind clinical trial comparing topical and sub-Tenon's anaesthesia in routine cataract surgery. $\mathrm{Br}$ Anaesth 2004; 93: 683-686.

7 Zafirakis P, Voudouri A, Rowe S, Livir-Rallatos G, Livir-Rallatos C, Canakis C et al. Topical vs sub-Tenon's anesthesia without sedation in cataract surgery. J Cataract Refract Surg 2001; 27: 873-879.

8 Eke T, Thompson JR. The National Survey of Local Anaesthesia for Ocular Surgery. II. Safety profiles of local anaesthesia techniques. Eye 1999; 13: 196-204.

9 Eke T, Thompson JR. Safety of local anaesthesia for cataract surgery: why we should look again. Eye 2003; 17: 127-128.

10 Gillow JT, Aggarwal RK, Kirkby GR. Ocular perforation during peribulbar anaesthesia. Eye 1996; 10: 533-536.

11 Grizzard WS, Kirk NM, Pavan PR, Antworth MV, Hammer ME, Roseman RL. Perforating ocular injuries caused by anesthesia personnel. Ophthalmology 1991; 98 1011-1015.

12 Hay A, Flynn HW, Hoffman JI, Rivera AH. Needle penetration of the globe during retrobulbar and peribulbar injections. Ophthalmology 1991; 98: 1017-1024.

13 Kumar CM. Orbital regional anesthesia: complications and their prevention. Indian J Ophthalmol 2006; 54: 77-84.

14 Kumar CM, Dowd TC. Complications of ophthalmic regional blocks: their treatment and prevention. Ophthalmologica 2006; 220: 73-82.

15 Roman SJ, Chong Sit DA, Boureau CM, Auclin FX, Ullern MM. Sub-Tenon's anaesthesia: an efficient and safe technique. Br J Ophthalmol 1997; 81: 673-676.

16 Stevens J. A new local anaesthesia technique for cataract extraction by one quadrant sub-Tenon's infiltration. $\mathrm{Br} \mathrm{J}$ Ophthalmol 1992; 76: 620-624.

17 Stevens JD. Curved sub-Tenon cannula for local anaesthesia Ophthalmic Surg 1993; 24: 121-123.

18 Verghese I, Sivraj P, Lai YK. The effectiveness of sub-Tenon's infiltration of local anaesthesia for cataract surgery. Aust N Z J Ophthalmol 1996; 24: 117-120.

19 Greenbaum S. Parabulbar anesthesia. Am J Ophthalmol 1992; 114: 776.

20 Kumar CM, Dodds C. A disposable plastic sub-Tenon cannula. Anaesthesia 2001; 56: 399-400.

21 Mather CM. Comparison of i.v. cannula and Stevens' cannula for sub-Tenon's block. Br I Anaesth 2007; 99: 421-424.

22 McNeela BJ, Kumar CM. Sub-Tenon's block with an ultrashort cannula. J Cataract Refract Surg 2004; 30: 858-862.

23 Allman KG, Theron AD, Byles DB. A new technique of incisionless minimally invasive sub-Tenon's anaesthesia. Anaesthesia 2008; 63: 782-783.

24 Vohra SB, Murray PI. Sub-tenon's block: a national United Kingdom survey. Ophthalmic Surg Lasers Imaging 2008; 393: 79-85.

25 Agency for Healthcare Research and Quality. Evidence Report/Technology Assessment: Number 16: Anaesthesia Management During Cataract Surgery. (Available at http:/ / www.ahcpr.gov/clinic/epcsums/anestsum.htm. Accessed on 26 October 2010)
26 Davison M, Padroni S, Bunce C, Rüschen H. Sub-Tenon's anaesthesia vs topical anaesthesia for cataract surgery. Cochrane Database Syst Rev 2007; (3): CD006291.

27 Lake AP, Puvanachandra K. Sub-tenon block: a learning curve of 100 cases. Whose benefit? Anaesth Intensive Care 2006; 34: 450-452.

28 Clarke JP, Roberton G, Plummer J. Sub-Tenon block: a learning curve of 100 cases. Anaesth Intensive Care 2006; 34: 825-826.

29 Kumar CM, Williamson S, Manickam B. A review of sub-Tenon's block: current practice and recent development. Eur J Anaesthesiol 2005; 22: 567-577.

30 Kumar CM, Dodds C. Sub-Tenon's anesthesia. Ophthalmol Clin North Am 2006; 19: 209-219.

31 Kumar C, Dodds C. Evaluation of Greenbaum's sub-Tenon's block. Br I Anaesth 2001; 87: 631-633.

32 Guise PA. Sub-Tenon anesthesia: a prospective study of 6,000 blocks. Anesthesiology 2003; 98: 964-968.

33 The Royal College of Anaesthetist and the Royal College of Ophthalmologists. Report on Local Anaesthesia for Intraocular Surgery 2001 (http:/ /www.rcoa.ac.uk/docs/ RCARCOGuidelines.pdf. Accessed on 26 October 2010).

34 Allen MJ, Bunce C, Presland AH. The effect of warming local anaesthetic on the pain of injection during sub-Tenon's anaesthesia for cataract surgery. Anaesthesia 2008; 63: 276-278.

35 Kwok AK, Van Newkirk MR, Lam DS, Fan DS. Sub-Tenon's anesthesia in vitreoretinal surgery: a needleless technique. Retina 1999; 19: 291-296.

36 Kumar CM, Dodds C, McLure H, Chabria R. A comparison of three sub-Tenon's cannulae. Eye 2004; 18: 873-876.

37 Kumar CM, Dowd TC, Adams WE, Puckering S. Methodology of evaluating conjunctival appearance following sub-Tenon's block for phacoemulsification cataract surgery. Eye 2006; 20: 1110-1111.

38 Kumar N, Jivan S, Thomas P, McLure H. Sub-Tenon's anesthesia with aspirin, warfarin, and chlopidogrel. Cataract Refract Surg 2006; 32: 1022-1025.

39 Koopmans SA, van Rij G. Cataract surgery and anticoagulants. Doc Ophthalmol 1996; 92: 11-16.

40 Konstantatos A. Anticoagulation and cataract surgery: a review of the current literature. Anaesth Intensive Care 2001; 29: $11-18$.

41 Assia EI, Raskin T, Kaiserman I, Rotenstreich Y, Segev F. Effect of aspirin intake on bleeding during cataract surgery. J Cataract Refract Surg 1998; 24: 1243-1246.

42 Gauba V, Saleh GM, Watson K, Chung A. Sub-Tenon anaesthesia: reduction in subconjunctival haemorrhage with controlled bipolar conjunctival cautery. Eye 2007; 21: 1387-1390.

43 Kumar CM, Dowd TC, Adams WE, Puckering S Methodology of evaluating conjunctival appearance following sub-Tenon's block for phacoemulsification cataract surgery. Eye 2006; 20: 1110-1111.

44 Ruschen H, Bremmer FD, Carr C. Complications after sub-Tenon's eye block. Anesthesia Analgesia 2003; 96: 273-277.

45 Standring S, Ellis H, Healy J, Johnson D, Williams A. In Gray's Anatomy: The Anatomical Basis of Clinical Practice, 40th edn. Churchill Livingston: London, 2008.

46 Quantock CL, Goswami T. Death potentially secondary to sub-Tenon's block. Anaesthesia 2007; 62: 175-177. 
47 Frieman BJ, Friedberg MA. Globe perforation associated with subtenon's anesthesia. Am J Ophthalmol 2001; 131: 520-521.

48 Faure C, Faure L, Billotte C. Globe perforation following no-needle sub-Tenon anesthesia. J Cataract Refract Surg 2009; 35: 1471-1472.

49 Ernest JT, Goldstick TK, Stein MA, Zheutlin JD. Ocular massage before cataract surgery. Trans Am Ophthalmol Soc 1985; 83: 205-217.

50 Olitsky SE, Juneja RG. Orbital hemorrhage after the administration of sub-Tenon's infusion anesthesia. Ophthalmic Surg Lasers 1997; 28: 145-146.

51 Rahman I, Ataullah S. Retrobulbar hemorrhage after subTenon's anesthesia. J Cataract Refract Surg 2004; 30: 2636-2637.

52 Subbiah S, McGimpsey S, Best RM. Retrobulbar hemorrhage after sub-Tenon's anesthesia. J Cataract Refract Surg 2007; 33: 1651-1652.

53 Goldsmith MO. Occlussion of the central retinal artery following retrobulbar hemorrhage. Ophthalmologica 1967; 153: 191-196.

54 Kraushar MF, Seelenfreund MH, Freilich DB. Central retinal artery closure during orbital hemorrhage from retrobulbar injection. Trans Am Acad Ophthalmol Otolaryngol 1974; 78: 65-70.

55 Klein ML, Jampol LM, Condon PI, Rice TA, Serjeant GR. Central retinal artery occlussion without retrobulbar hemorrhage after retrobulbar anesthesia. Am J Ophthalmol 1982; 93: 573-577.

56 Feibel RM, Guyton DL. Transient central retinal artery occlusion after posterior sub-Tenon's anesthesia. J Cataract Refract Surg 2003; 29: 1821-1824.

57 Fry RA, Ring P. Cilioretinal artery occlusion associated with sub-Tenon's regional blockade. Clin Experiment Ophthalmol 2008; 36: 196-197.

58 Alwitry A, Koshy Z, Browning AC, Kiel W, Holden R. The effect of sub-Tenon's anaesthesia on intraocular pressure. Eye 2001; 15: 733-735.

59 Pianka P, Weintraub-Padova H, Lazar M, Geyer O. Effect of sub-Tenon's and peribulbar anesthesia on intraocular pressure and ocular pulse amplitude. J Cataract Refract Surg 2001; 27: 1221-1226.

60 Watkins R, Beigi B, Yates M, Chang B, Linardos E. Intraocular pressure and pulsitile ocular blood flow after retrobulbar and peribulbar anaesthesia. $\mathrm{Br} J$ Ophthalmol 2001; 85: 796-798.

61 Meyer P, Flammer J, Lüscher TF. Local anesthetic drugs reduce endothelium-dependent relaxations of porcine ciliary arteries. Invest Ophthalmol Vis Sci 1993; 34: 2730-2736.

62 Kim SK, Andreoli CM, Rizzo III JF, Golden MA, Bradbury MJ. Optic neuropathy secondary to sub-tenon anesthetic injection in cataract surgery. Arch Ophthalmol 2003; 121: 907-909.

63 Jaycock PD, Mather CM, Ferris JD, Kirkpatrick JN. Rectus muscle trauma complicating sub-Tenon's local anaesthesia. Eye 2001; 15: 583-586.
64 Adams W, Morgan SJ. Diplopia following sub-Tenon's infiltration of local anesthesia. J Cataract Refract Surg 2002; 28: 1694-1697.

65 Merino P, Muñoz-Sanz N, Gómez-de-Liaño P, GutiérrezPartida B, Seijas-Leal O. Diplopia after sub-Tenon's anesthesia for cataract surgery. Arch Soc Esp Oftalmol 2006; 81: 141-146.

66 Spierer A, Schwalb E. Superior oblique muscle paresis after sub-Tenon's anesthesia for cataract surgery. J Cataract Refract Surg 1999; 25: 144-145.

67 Lip PL, Moutsou M, Hero M. A post-operative complication far worse than endophthalmitis: the co-existing of orbital cellulitis. Br J Ophthalmol 2001; 85: 631-632.

68 Redmill B, Sandy C, Rose GE. Orbital cellulitis following corneal gluing under sub-Tenon's local anaesthesia. Eye 2001; 15: 554-556.

69 Dahlmann AH, Appaswamy S, Headon MP. Orbital cellulitis following sub-tenon's anaesthesia. Eye 2002; 16: 200-201.

70 Morgan SJ. Orbital cellulitis following corneal gluing under sub-Tenon's local anaesthesia. Eye 2003; 17: 284-285.

71 Kumar CM, Dowd TC, Dodds C, Boyce R. Orbital swelling following peribulbar and sub-Tenon's anaesthesia. Eye 2004; 18: 418-420.

72 Mukherji S, Esakowitz L. Orbital inflammation after subTenon's anesthesia. J Cataract Refract Surg 2005; 31: 2221-2223.

73 Liang SY, Moloney G, O'Donnell BA, Fernando G. Orbital cellulitis as a postoperative complication of sub-Tenon anaesthesia in cataract surgery. Clin Experiment Ophthalmol 2006; 34: 897-899.

74 Quhill F, Bowling B, Packard RB. Hyaluronidase allergy after peribulbar anesthesia with orbital inflammation. J Cataract Refract Surg 2004; 30: 916-917.

75 Ciulla TA, Starr MB, Masket S. Bacterial endophthalmitis prophylaxis for cataract surgery: An evidence-based update. Ophthalmology 2003; 109: 13-24.

76 British National Formulary. A joint publication of the British National Formulary (British Medical Association) and the Royal Pharmaceutical Society of Great Britain. British Medical Journal Publishing Group: London, 2009.

77 Costen MT, Bolton K, Boase DL. A lash foreign body complicating subtenon's anaesthesia. Eye 2004; 18: 192-193.

78 Aslam SA, Jayaram H, Ali N. Sub-Tenon's block complicated by subconjunctival cilia. J Cataract Refract Surg 2007; 33: 1490-1491.

79 Heatley CJ, Marshall J, Toma M. A trip to eye casualty an unusual complication of sub-Tenon's anaesthesia. Eye 2006; 20: 738-739.

80 Vishwanath MR, Jain A. Conjunctival inclusion cyst following sub-Tenon's local anaesthetic injection. $\mathrm{Br} J$ Anaesth 2005; 95: 825-826.

81 Kumar CM. Low volume sub-Tenon's block. Anaesthesia 2007; 62: 970-971. 